\title{
Old stellar population synthesis: New age and mass estimates for Mayall II = G1
}

\author{
Jun $\mathrm{Ma}^{1 \star}$, Richard de Grijs ${ }^{2,1}$, Zhou Fan ${ }^{1,3}$, Soo-Chang Rey ${ }^{4}$, Zhenyu Wu ${ }^{1}$, Xu Zhou ${ }^{1}$, \\ Jianghua Wu ${ }^{1}$, Zhaoji Jiang ${ }^{1}$, Jiansheng Chen ${ }^{1}$, Kyungsook Lee ${ }^{4}$ and S. T. Sohn ${ }^{5,6}$ \\ 1 National Astronomical Observatories, Chinese Academy of Sciences, Beijing, 100012, P. R. China \\ 2 Department of Physics \& Astronomy, The University of Sheffield, Hicks Building, Hounsfield Road, \\ Sheffield S3 7RH, UK \\ 3 Graduate University, Chinese Academy of Sciences, Beijing, 100039, P. R. China \\ 4 Department of Astronomy and Space Science, Chungnam National University, Daejeon 305-764, \\ Korea \\ ${ }^{5}$ Center for Space Astrophysics, Yonsei University, Seoul 120-749, Korea \\ ${ }^{6}$ California Institute of Technology, MC 405-47, 1200 E. California Boulevard, Pasadena, CA 91125
}

\begin{abstract}
Mayall II = G1 is one of the most luminous globular clusters (GCs) in M31. Here, we determine its age and mass by comparing multicolor photometry with theoretical stellar population synthesis models. Based on far- and near-ultraviolet GALEX photometry, broad-band $U B V R I$, and infrared $J H K_{\mathrm{s}}$ 2MASS data, we construct the most extensive spectral energy distribution of G1 to date, spanning the wavelength range from 1538 to $20,000 \AA$. A quantitative comparison with a variety of simple stellar population (SSP) models yields a mean age that is consistent with G1 being among the oldest building blocks of M31 and having formed within $~ 1.7$ Gyr after the Big Bang. Irrespective of the SSP model or stellar initial mass function adopted, the resulting mass estimates (of order $10^{7} M_{\odot}$ ) indicate that G1 is one of the most massive GCs in the Local Group. However, we speculate that the cluster's exceptionally high mass suggests that it may not be a genuine GC. We also derive that G1 may contain, on average, $(1.65 \pm 0.63) \times 10^{2} L_{\odot}$ far-ultraviolet-bright, hot, extreme horizontal-branch stars, depending on the SSP model adopted. On a generic level, we demonstrate that extensive multi-passband photometry coupled with SSP analysis enables one to obtain age estimates for old SSPs to a similar accuracy as from integrated spectroscopy or resolved stellar photometry, provided that some of the free parameters can be constrained independently.
\end{abstract}

Key words: galaxies: individual (M31) - galaxies: star clusters - galaxies: stellar content

\section{INTRODUCTION}

Globular clusters (GCs) are among the oldest bound stellar systems in the Universe, and they thus provide a fossil record of the earliest stages of galaxy formation and evolution. GCs are internally homogeneous in age and metallicity, i.e. they are simple stellar systems composed of coeval stellar populations. In addition, GCs are the oldest systems in our own and other galaxies for which we can estimate reasonably reliable ages (and realistic uncertainties); they can thus independently provide vitally important information regarding the minimum age of the Universe. In a detailed study of 17 Galactic

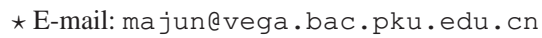


GCs, Chaboyer et al. (1998) used the improved Hipparcos parallaxes having just become available at that time to determine updated distances, and hence improved ages, of their GC sample. They concluded that the mean age of the oldest GCs is $11.5 \pm 1.3 \mathrm{Gyr}$, although their age histogram (their fig. 2) shows a tail toward ages as old as $\sim 16$ Gyr. Gratton et al. (2003) obtain improved ages (and distances) for three Galactic GCs, NGC 6397, NGC 6752, and 47 Tuc, and conclude that the age of the oldest GCs is $13.4 \pm 0.8$ (random) \pm 0.6 (systematic) Gyr, in good agreement with the 3 -year results from the Wilkinson Microwave Anisotropy Probe (WMAP). This led them to suggest that the oldest Galactic GCs formed within the first 1.7 Gyr after the Big Bang, at the $1 \sigma$ confidence level. We note that this is still fully compatible with the 5-year WMAP results constraining the age of the Universe to $13.73 \pm 0.12$ Gyr (Hinshaw et al. 2008). While the ages of the oldest GCs in the Galaxy are now reasonably well determined, this is certainly not the case even for our nearest large neighbour, the Andromeda galaxy (M31).

The most direct method for determining the age of a star cluster is by means of individual stellar photometry, since the main-sequence turn-off location is mostly affected by age (see, e.g., Puzia et al. 2002 b, and references therein). However, this method has only been applied to Galactic GCs and to GCs associated with the Milky Way's satellites (e.g., Riich 2001), in which individual stars can be resolved and their photometry determined to satisfactory accuracy, to a few magnitudes fainter than the main-sequence turn-offs. This is difficult, if not impossible, to achieve even for GCs as close as those associated with M31 (but see Brown et al. 2004), at a distance of $D=772 \pm 44 \mathrm{kpc}$ (e.g., Ribas et al. 2005). Fortunately, starting from the pioneering work of Tinsley $(1968,1972)$ and Searle et al. (1973), evolutionary population synthesis modeling has become a powerful tool to address many outstanding problems in astrophysics, from determining the ages of star clusters to interpreting integrated spectrophotometric observations of galaxies. Therefore, extragalactic GC ages can, in general, also be inferred from composite colors and/or integrated spectroscopy.

The evolution of GCs is usually modeled by means of the simple stellar population (SSP) approximation. An SSP is a single generation of coeval stars formed from the same progenitor molecular cloud (thus implying a single metallicity), and governed by a given stellar initial mass function (IMF). GCs are ideal templates to test the compatibility between the population synthesis models and reality. Barmby \& Huchra (2000) compared the predicted SSP colors of three stellar population synthesis models to the intrinsic broad-band $U B V R I J H K$ colors of Galactic and M31 GCs, and concluded that the best-fitting models match the cluster colors very well. Subsequently, many authors have used SSP modeling to determine the parameters of cluster populations. For instance, de Grijs et al. (2003a) determined the ages and masses of star clusters in the fossil starburst region B of M82 by comparing the observed cluster spectral energy distributions (SEDs) with both the STARBURST99 SSP models (Leitherer et al. 1999) and those developed by Bruzual \& Charlot (2000), based on Hubble Space Telescope (HST) observations from the blue optical to the near-infrared (NIR) (see also de Grijs et al. 2003b, 2003c); Bik et al. (2003) and Bastian et al. (2005) estimated ages, initial masses and extinction values for M51 star cluster candidates by comparing the STARBURST99 and the Frascati models (Romaniello 1998) for instantaneous star formation with the observed SEDs based on HST/WFPC2 observations in six broadband and two narrow-band filters. Ma et al. (2006) and Fan et al. (2006) obtained age estimates for M31 GCs by fitting theoretical stellar population synthesis models (Bruzual \& Charlot 2003, henceforth $\mathrm{BC} 03$ ) to their photometric measurements in a large number of intermediate- and broad-band passbands from the optical to the NIR. Based on the same method and models, Ma et al. (2007a) constrained the age of the M31 GC S312, using multicolor photometry from the near-ultraviolet (NUV) to the NIR, to $9.5_{-0.99}^{+1.15}$ Gyr. S312 is among the first extragalactic GCs for which the age was estimated accurately using main-sequence photometry, i.e., Brown et al. (2004) estimated the age of S312 at $10_{-1}^{+2.5}$ Gyr by means of a quantitative comparison with the isochrones of VandenBerg et al. (2006). This was based on their analysis of the cluster's color-magnitude diagram (CMD) below the main-sequence turn-off using extremely deep images obtained with the HST/Advanced Camera for Surveys (ACS).

It is a common misconception that spectroscopic age estimates are always much better than those based on broad-band photometry. Schweizer et al. (2004) recently showed convincingly that spectro- 
scopic age determinations are not necessarily better or more accurate than photometrically obtained ages, at least in the age range from $\sim 100-500$ Myr. Anders et al. (2004) published a detailed theoretical investigation of the accuracy of retrieved star cluster properties, including their ages, based on sophisticated fits of SSP models to observed broad-band SEDs spanning varying wavelength ranges. They concluded that if one has access to as large a wavelength range as possible, ideally including both ultraviolet and NIR data points, the resulting age estimates are reasonably accurate, even for ages as old as $\sim 10 \mathrm{Gyr}-$ particularly if one or more of the other free parameters (e.g., metallicity or extinction) can be constrained reliably and independently (see Anders et al. 2004, e.g., their fig. 14). We will use this promising approach as our basic premise in this paper.

Of the Local Group members, M31 is particularly important as it provides a direct comparison with our own Galaxy. In addition, it contains a large number of GCs and GC candidates, including 496 genuine, 367 probable, and 301 possible GCs (Kim et al. 2007). The M31 GC system is among the extragalactic GC systems studied most often (Harris 1991; Brodie \& Strader 2006). As one of the brightest M31 GCs, Mayall II = G1 has attracted much scientific interest (see, e.g., Barmby et al. 2007; Ma et al. 2007b, and references therein).

In this paper, we first determine the age and mass of G1 by comparing observational SEDs ( $(2)$ with population synthesis models $(\S 3)$. We will use the lessons learned from studies of broad-band photometric SED fits to minimize the associated uncertainties. We discuss our results along the way, as appropriate, and provide a summary in $\S 4$.

\section{ULTRAVIOLET, OPTICAL, AND INFRARED OBSERVATIONS OF G1}

\subsection{Historical observations}

G1 was first detected by Mayall \& Eggen (1953) (their No. 2, and hence referred to as Mayall II), who searched for nebulous objects associated with M31 using a $6^{\circ} \times 6^{\circ}$ Palomar 48-inch Schmidt plate taken in 1948 and centered on M31. Subsequently, Sargent et al. (1977) rediscovered the cluster (their No. 1, i.e., G1) based on their survey of 29 plates associated with the general field of M31, which had been obtained at the $f / 2.7$ prime focus of the Kitt Peak National Observatory's (KPNO) 4-m telescope. The cluster is located in the halo of M31, at a projected distance of about $40 \mathrm{kpc}$ from the galaxy's nucleus (see Meylan et al. 2001).

\subsection{GALEX ultraviolet, optical broad-band, and 2MASS NIR photometry}

Although the cluster is generally believed to be among the oldest GCs in M31, to the best of our knowledge there is no CMD-based or spectroscopic age estimate available in the literature to date. The lack of a CMD-based age estimate is due to the challenges associated with probing the cluster's CMD down to below the main-sequence turn-off. The current deepest CMD of the cluster (Meylan et al. 2001) does not reach these faint levels. Although both integrated and spatially-resolved spectra of the cluster are available (e.g., Huchra et al. 1991; Gebhardt et al. 2005; Cohen 2006), they have thus far not been used to determine an age for G1. This may be partially due to the limited wavelength range covered by most of these spectra, and the difficulties one faces when trying to constrain ages in the regime beyond $\sim 10$ Gyr (see below).

To constrain the age of G1 accurately, with the smallest uncertainty allowed by the observational data, we use as many photometric data points covering as large a wavelength range as possible. Kaviraj et al. (2007) showed that the combination of far (FUV) and near-ultraviolet photometry with optical observations in the standard broad bands enables one to efficiently break the age-metallicity degeneracy; Worthey (1994) showed that the age-metallicity degeneracy associated with optical broadband colors is $\Delta$ age/ $\Delta Z \sim 3 / 2$ (also see MacArthur et al. 2004). However, de Jong (1996) showed that this degeneracy can be partially broken by adding NIR photometry to the optical colors, which was recently supported by Wu et al. (2005). Cardiel et al. (2003) found that inclusion of an infrared passband can improve the predictive power of the stellar population diagnostics by $\sim 30$ times compared to 
Table 1 Ultraviolet, optical broad-band, and NIR 2MASS photometry of G1.

\begin{tabular}{lcc}
\hline Filter & Magnitude (uncertainty) & Reference \\
\hline FUV & $18.972(0.031)$ & Rey et al. (2007) \\
NUV & $18.014(0.012)$ & \\
$U$ & $14.85(0.08)$ & van den Bergh (1969) \\
& & \\
$B$ & $14.584(0.013)$ & Reed, Harris \& Harris (1994) \\
$R$ & $13.750(0.007)$ & \\
$I$ & $13.191(0.010)$ & Barmby \& Huchra (2001) \\
$J$ & $12.684(0.060)$ & Galleti et al. (2004) \\
$H$ & $11.858(0.054)$ & \\
$K_{\mathrm{s}}$ & $11.127(0.054)$ & \\
\hline
\end{tabular}

using optical photometry alone. Since NIR photometry is less sensitive to interstellar extinction than the classical optical passbands, Kissler-Patig et al. (2002) and Puzia et al. (2002a) also suggested that it provides useful complementary information that can help to disentangle the age-metallicity degeneracy (also see Galleti et al. 2004).

The M31 field was observed as part of the Nearby Galaxies Survey (NGS) by the Galaxy Evolution Explorer (GALEX) in two ultraviolet bands (see for details from Rey et al 2005, 2007). Rey et al. (2007) published photometric data for 485 and 273 M31 GCs in the GALEX NUV and FUV bands, respectively. G1 was detected in these two ultraviolet bands. The GALEX photometric system is calibrated to match the spectrophotometric AB system.

van den Bergh (1969) determined photo-electric photometry for 45 M31 GCs, including G1, in the $U B V$ bands. Using CCD imaging from the KPNO 0.9m telescope, Reed, Harris \& Harris (1994) published integrated $B V R$ magnitudes and color indices for $41 \mathrm{GCs}$ and GC candidates, including $\mathrm{G} 1$, in the outer halo of M31. We compared the photometry of G1 in the $B$ and $V$ bands between these two studies; the results match closely. In this paper, we adopt the CCD $B V R$ photometry of Reed, Harris \& Harris (1994), and the photographic $U$-band photometry of van den Bergh (1969), with a photometric uncertainty of $0.08 \mathrm{mag}$ as suggested by Galleti et al. (2004). Based on HST images, Barmby \& Huchra (2001) detected and published photometry for 114 GC candidates associated with M31, including 32 new objects. Their $V$-band photometry is in good agreement with that of van den Bergh (1969) and Reed, Harris \& Harris (1994), although they do not provide their photometric uncertainties. However, Barmby \& Huchra (2001) compared their HST photometry with the ground-based measurements compiled by Barmby et al. (2000), and found that the median offset in $I$ is $0.06 \pm 0.04$ mag. Therefore, we adopt $0.06 \mathrm{mag}$ as the photometric uncertainty in the $I$ band. Using the Two Micron All Sky Survey (2MASS) database, Galleti et al. (2004) identified 693 known and candidate GCs in M31, and listed their 2MASS $J H K_{\mathrm{S}}$ magnitudes. Galleti et al. (2004) transformed all 2MASS magnitudes to the CIT photometric system (Elias et al. 1982, 1983) using the color transformations in Carpenter (2001). However, we need the original 2MASS $J H K_{\mathrm{s}}$ magnitudes to compare our observational SEDs with the SSP models, so we reversed this transformation using the same procedures. Since Galleti et al. (2004) do not provide the photometric uncertainties in $J H K_{\mathrm{s}}$, we obtained these by comparing the magnitudes with fig. 2 of Carpenter et al. (2001), where the observed photometric rms uncertainties in the time series are shown as a function of magnitude, for stars brighter than the observational completeness limits. In fact, the photometric uncertainties adopted do not affect our results significantly, as we showed in Fan et al. (2006) (see their section 4.3 for details). The full set of ultraviolet, optical broad-band, and 2MASS NIR photometry of G1 is listed in Table 1. The $U B V R I$ and 2MASS magnitudes are given in the Vega system Schneider et al. (1977). For convenience, we converted all observational magnitudes to the $\mathrm{AB}$ system, following the procedures recommended in $\mathrm{BC} 03$. 


\subsection{Reddening and metallicity}

To obtain the intrinsic SED of G1, the photometric data must first be dereddened. Since G1 is located in the halo of M31, i.e., far away from the galaxy's disk, it is (for all practical purposes) only affected by Galactic foreground extinction. In fact, some authors have demonstrated that G1 is affected by a negligible amount of reddening. Mevlan et al. (2001) used HST/WFPC2 observations in the F555W and F814W filters, and applied Sarajedini (1994)'s method to simultaneously determine the cluster's reddening and metallicity; they obtained a reddening of $E(V-I)=0.05 \pm 0.02$ mag toward G1, which is less than the Galactic foreground extinction. van den Bergh (1969) studied the reddening in the halo of M31 by comparing the colors of clusters with the same line-strength index in the Galaxy and in M31, and obtained a mean reddening of $E(B-V)=0.08 \pm 0.02$ mag for the clusters in the halo of M31. Barmby et al. (2000) determined the reddening for each individual cluster using correlations between optical and infrared colors and metallicity, and by defining various 'reddening-free' parameters based on their large database of multi-color photometry. For G1, Barmby et al. (2000, also P. Barmby, priv. comm.) obtained $E(B-V)=0.09 \pm 0.02 \mathrm{mag}$. In this paper, we adopt the reddening value from Barmby et al. (2000). The values for the extinction coefficient, $R_{\lambda}$, were obtained by interpolating the interstellar extinction curve of Cardelli et al. (1989).

Cluster SEDs are determined by the combination of their ages and metallicities, which is often referred to as the age-metallicity degeneracy. Therefore, the age of a cluster can only be constrained accurately if the metallicity is known with confidence, from independent determinations. There exist two metallicity determinations for G1. Huchra et al. (1991) derived metallicities for 150 M31 GCs, including G1, using the strengths of six absorption features in the clusters' integrated spectra. The resulting metallicity of G1 is $[\mathrm{Fe} / \mathrm{H}]=-1.08 \pm 0.09$. Meylan et al. (2001) used HST/WFPC2 photometry to construct deep CMDs for G1, combined with the shape of the red-giant branch as calibrated by Saraiedini et al. (2000), to derive the mean metallicity of G1 on the scale of Zinn \& West (1984), $[\mathrm{Fe} / \mathrm{H}]=-0.95 \pm 0.09$. In this paper, we adopt $[\mathrm{Fe} / \mathrm{H}]=-1.08 \pm 0.09$ for $\mathrm{G} 1$.

\section{THE STELLAR POPULATION OF G1}

\subsection{Stellar populations and synthetic photometry}

In this section, we compare the SED of G1 with theoretical stellar population synthesis models. We start by using the BC03 SSP models, which have been upgraded from the earlier Bruzual \& Charlot (1993, 1996) versions, and now provide the evolution of the spectra and photometric properties for a wider range of stellar metallicities. BC03 provide 26 SSP models (both of high and low spectral resolution) using the Padova-1994 evolutionary tracks, half of which were computed based on the Salpeter (1955) IMF with lower and upper-mass cut-offs of $m_{\mathrm{L}}=0.1 M_{\odot}$ and $m_{\mathrm{U}}=100 M_{\odot}$, respectively. The other thirteen were computed using the Chabrier (2003) IMF with the same mass cut-offs. In addition, BC03 provide 26 SSP models using the Padova-2000 evolutionary tracks. In this paper, we will use all of these SSP models to determine the most appropriate age and mass for G1. These SSP models contain 221 spectra describing the spectral evolution of SSPs from $1.0 \times 10^{5} \mathrm{yr}$ to $20 \mathrm{Gyr}$. The evolving spectra include the contribution of the stellar component at wavelengths from $91 \AA$ to $160 \mu \mathrm{m}$.

Since our observational data are integrated luminosities through a given set of filters, we convolved the theoretical SSP SEDs of BC03 with the FUV and NUV, broad-band $U B V R I$, and 2MASS $J H K_{\mathrm{s}}$ filter response curves to obtain synthetic ultraviolet, optical, and NIR photometry for comparison. The synthetic magnitude in the $\mathrm{AB}$ magnitude system for the $i$ th filter can be computed as

$$
m_{\lambda_{i}}=-2.5 \log \frac{\int_{\lambda} F_{\lambda} \varphi_{i}(\lambda) \mathrm{d} \lambda}{\int_{\lambda} \varphi_{i}(\lambda) \mathrm{d} \lambda}-48.60
$$

where $F_{\lambda}$ is the theoretical SED and $\varphi_{i}$ the response curve of the $i$ th filter. $F_{\lambda}$ varies as a function of age and metallicity. 


\subsection{Fit results}

We use a $\chi^{2}$ minimization approach to examine which SSP models are most compatible with the observed SEDs, following

$$
\chi^{2}=\sum_{i=1}^{10} \frac{\left[m_{\lambda_{i}}^{\mathrm{intr}}-m_{\lambda_{i}}^{\bmod }(t)\right]^{2}}{\sigma_{i}^{2}},
$$

where $m_{\lambda_{i}}^{\bmod }(t)$ is the integrated magnitude in the $i$ th filter of a theoretical SSP at age $t, m_{\lambda_{i}}^{\text {intr }}$ represents the intrinsic integrated magnitude in the same filter, and

$$
\sigma_{i}^{2}=\sigma_{\mathrm{obs}, i}^{2}+\sigma_{\bmod , i}^{2}
$$

Here, $\sigma_{\mathrm{obs}, i}^{2}$ is the observational uncertainty, and $\sigma_{\text {mod, } i}^{2}$ is the uncertainty associated with the model itself, for the $i$ th filter. Charlot et al. (1996) estimated the uncertainty associated with the term $\sigma_{\bmod , i}^{2}$ by comparing the colors obtained from different stellar evolutionary tracks and spectral libraries. Following Wu et al. (2005), we adopt $\sigma_{\bmod , i}^{2}=0.05$.

The BC03 SSP models based on the Padova-1994 evolutionary tracks include six initial metallicities, $Z=0.0001,0.0004,0.004,0.008,0.02\left(Z_{\odot}\right)$, and 0.05 , corresponding to $[\mathrm{Fe} / \mathrm{H}]=$ $-2.25,-1.65,-0.64,-0.33,+0.09$, and +0.56 . However, the BC03 SSP models based on the Padova2000 evolutionary tracks include six partially different initial metallicities, $Z=0.0004,0.001,0.004$, $0.008,0.019\left(Z_{\odot}\right)$, and 0.03 , i.e., $[\mathrm{Fe} / \mathrm{H}]=-1.65,-1.25,-0.64,-0.33,+0.07$, and +0.29 . Spectra for other metallicities can, in principle, be obtained by interpolation of the appropriate spectra for any of these metallicities, although this is not necessarily advisable or straightforward (Frayn \& Gilmore 2002). Instead, we adopt the most appropriate model metallicity for the analysis performed in this paper. Since we have good estimates of the metallicity and reddening values of G1 (see $\$ 2.3$ ), the cluster age is therefore the sole parameter to be estimated (for a given IMF and extinction law, both of which we assume to be universal).

None of the SSP models fit the photometric data point in the GALEX FUV band as well as the other nine data points. (We checked that the image of G1 in the FUV band is not affected by instrumental problems.) Given that G1 contains an old stellar population, the most likely physical explanation for this FUV excess compared to the 'standard' BC03 SSP models is the presence of a significant number of FUV-bright, hot, extreme horizontal-branch (EHB) stars giving rise to the well-known 'ultraviolet upturn' below $\lambda \simeq 2000 \AA$ (see, e.g., the review of O'Connell 1999, and references therein; see also Landsman et al. 1998; Sohn et al. 2006). (Alternative species, such as AGB-manqué stars or blue stragglers are expected to be fewer in number in any 'normal' stellar population.)

Since 'standard' SSP models do not contain EHB populations, we are forced to deselect the photometric FUV data point when applying our fitting routines. In Fig. 1, we show the intrinsic SED of G1 and the integrated SEDs of the best-fitting models. The dereddened data are shown as the symbols with error bars (vertical errors for photometric uncertainties and horizontal error bars for the approximate wavelength coverage of each filter); open circles represent the calculated magnitudes of the model SED for each filter, obtained by convolving the theoretical SSP SEDs with the appropriate filter response curves. The best reduced- $\chi^{2}$ values and ages are listed in Table 2. The mass of G1, also listed in Table 2, can be estimated by comparing the measured luminosity in the $V$ band with the theoretical mass-tolight $(M / L)$ ratios. These $M / L$ ratios are a function of the cluster age and metallicity. The mass-to-light ratios of G1, calculated based on the metallicity adopted and the age obtained in this paper, are listed in Table 2 for the BC03 SSP models. Based on its present luminosity, $V=13.750 \pm 0.007 \mathrm{mag}$, and extinction, $E(B-V)=0.09 \mathrm{mag}$, the cluster's visual magnitude corrected for the extinction is $V_{0}=13.471 \pm 0.007 \mathrm{mag}$, assuming a Cardelli et al. (1989) Galactic reddening law with $A_{V}=0.279$ mag.

(We note that the NUV data point is also marginally affected by the onset of the UV upturn, which causes a slight mismatch between the observations and the best-fitting theoretical SSP models.) 

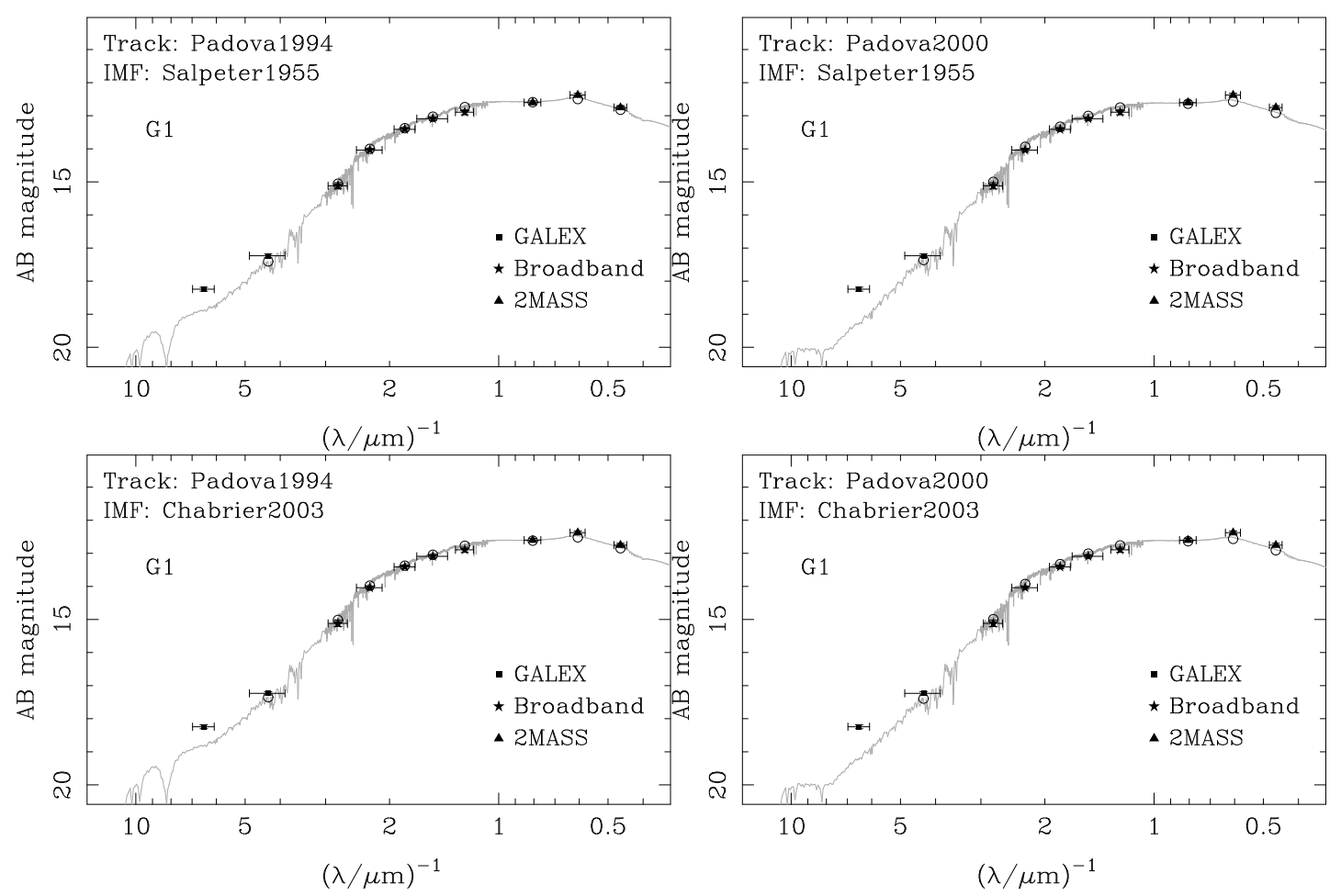

Fig. 1 Best-fitting integrated theoretical BC03 SEDs compared to the intrinsic SED of G1. The photometric measurements are shown as the symbols with error bars (vertical for uncertainties and horizontal for the approximate wavelength coverage of each filter). Open circles represent the calculated magnitudes of the model SED for each filter. We did not use the FUV photometric data point for the fits (see text).

\subsection{Age and mass}

In the previous section we determined the best-fitting age and mass of G1 based on different theoretical SSP models. From Table 2 we conclude that, within the errors, the ages obtained from the different BC03 models are internally consistent. The mean age of G1 is $18.23 \pm 1.76$ Gyr. This is in excellent agreement with the only other available (rough) age estimate for the cluster by Meylan et al. (2001), who estimated its age to be $\sim 15 \mathrm{Gyr}$. However, we note that the age of G1 obtained in this paper is older than the current-best estimate of the age of the Universe, of order $13.7 \mathrm{Gyr}$, as discussed in $\S 3.1$. We will discuss this problem in $\S 4$.

We conclude that the various mass estimates listed in Table 2 place G1 firmly at the top of the cluster mass function in the Local Group. Meylan et al. (2001) presented three estimates of the total mass of G1, (i) a King-model mass (King 1966) of $1.5 \times 10^{7} M_{\odot}$, (ii) a virial mass of $0.75 \times 10^{7} M_{\odot}$; and (iii) a mass based on a King-Michie model (as defined by Gunn \& Griffi 1979) fitted simultaneously to the surface brightness profile and the central velocity dispersion value, estimated between $1.4 \times 10^{7} M_{\odot}$ and $1.7 \times 10^{7} M_{\odot}$. Our results are in reasonable agreement with Mevlan et al. (2001), although we are aware that the King and King-Michie mass estimates of Meylan et al. (2001) are up to a factor of two greater than our photometric mass estimates. This is not too surprising in view of the model assumptions made. Cohen (2006) recently obtained an optical velocity dispersion for the cluster using the Keck/HIRES spectrograph, and derived an aperture-corrected line-of-sight velocity dispersion, $\sigma_{\text {los }}=25.5 \pm 1.5 \mathrm{~km}$ $\mathrm{s}^{-1}$ (where we have averaged the values she obtained for the two reddest wavelength ranges analyzed; 
Table 2 Age and mass estimates of G1 based on the BC03 models.

\begin{tabular}{cccccc}
\hline Evolutionary Track & \multicolumn{1}{c}{ IMF } & $\begin{array}{c}\text { Age } \\
(\mathrm{Gyr})\end{array}$ & $\chi^{2} /$ degree of freedom & $\begin{array}{c}M / L_{V} \\
\left(M / L_{V}\right) \odot\end{array}$ & $\begin{array}{c}\text { Mass } \\
\left(10^{7} M_{\odot}\right)\end{array}$ \\
\hline Padova 1994 & Salpeter (1955) & $19.68 \pm 0.75$ & 3.04 & 5.10 & $1.06 \pm 0.07$ \\
Padova 1994 & Chabrier (2003) & $19.79 \pm 0.50$ & 2.69 & 3.15 & $0.65 \pm 0.04$ \\
Padova 2000 & Salpeter (1955) & $15.44 \pm 0.78$ & 5.36 & 4.14 & $0.86 \pm 0.05$ \\
Padova 2000 & Chabrier (2003) & $18.01 \pm 2.00$ & 5.27 & 2.79 & $0.58 \pm 0.04$ \\
\hline
\end{tabular}

see also Djorgovski et al. 2002). We recently redetermined a projected half-light radius for G1 of $r_{\mathrm{h}}=$ $6.5 \pm 0.3 \mathrm{pc}$ (Ma et al. 2007b). Thus, based on these most recent results, the dynamical (virial) mass of G1 is $M_{\text {vir }}=(7.37 \pm 2.15) \times 10^{6} M_{\odot}$. This is in excellent agreement with the photometric mass estimates obtained in this paper. In turn, this strongly supports the notion that G1 must have had a closeto-'normal' stellar IMF, in order for it to have survived dissolution due to internal two-body relaxation until the present time (see also Ma et al. 2006). In particular, this is driven by the observation that if the IMF is too shallow, i.e., if a cluster is significantly depleted in low-mass stars compared to (for instance) the solar neighborhood, it will disperse within a few orbital periods around its host galaxy's center, and most likely within about a Gyr of its formation (e.g., Gnedin \& Ostriker 1997; Goodwin 1997; Smith \& Gallagher 2001; Mengel et al. 2002; Rose, Kouwenhoven \& de Grijs, in prep.).

From the recent work of Ma et al. (2006), the intrinsically most luminous M31 GC, 037-B327, has been suggested to be the most massive GC in the Local Group, with a total mass of $\sim(3.0 \pm$ $0.5) \times 10^{7} M_{\odot}$, also determined photometrically and somewhat depending on the SSP models used, the metallicity and age adopted, and the IMF representation. However, Cohen (2006) pointed out that the photometric mass of this cluster had likely been overestimated due to an incorrect extinction correction. Nevertheless, she also confirmed the nature of 037-B327 as one of the most massive GCs in the Local Group, with a dynamical mass similar to that of G1. It is intriguing that these two most massive GC in M31 both are significantly more massive than the most massive Galactic GC, $\omega$ Cen $[\sim(2.9-5.1) \times$ $10^{6} M_{\odot} ;$ Meylan (2002)].

In fact, the high mass of these clusters raises additional, intriguing questions regarding the nature of these objects in general, and of G1 in particular (see also Federici et al. 2007; Ma et al. 2007b, and references therein). It has been speculated that these objects may be nucleated dwarf galaxies instead of genuine GCs. In support of this notion, we point out that Gieles et al. (2006) suggest that there may be a physical upper limit to the mass of a star cluster that is not merely the result of size-of-sample effects. This maximum mass depends to some extent on the galactic environment; for their example galaxies, M51 and the Antennae system, they find a physical upper limit to the stellar mass of $\sim\left(10^{5}-10^{6}\right) M_{\odot}$. Our values derived for both the photometric and the virial mass of G1 are well above these suggested upper mass limits. This may, therefore, provide an additional (although circumstantial) proverbial nail in the coffin of G1 as a normal GC.

\subsection{Luminosity of the hot, extreme horizontal-branch stars}

As discussed in $\S 3.3$, none of the BC03 SSP models fit the photometric data point in the GALEX FUV band as well as the other nine data points. EHB stars may be responsible for this excess in FUV band. In fact, Rich et al. (1996) found some bluer horizontal-branch stars extending to $(V-I)=0.0$ mag, based on their HST/WFPC2 observations. In this section, we will calculate the luminosity of the EHB stars. We assume that the excess in the GALEX FUV band is solely due to these EHB stars. The magnitude differences between the four SSP models employed in this paper and the photometric data points are $0.63,1.00,0.56$, and $0.94 \mathrm{mag}$, respectively, corresponding $(2.44,1.07,2.11$, and 1.00$) \times$ $10^{2} L_{\odot}$, respectively, with a mean number of EHB stars in G1 of $(1.65 \pm 0.63) \times 10^{2} L_{\odot}$. 


\subsection{Comparison between G1 and S312}

Brown et al. (2004) showed that a 10 Gyr old population in M31 has a main-sequence turnoff at about $m_{\mathrm{F} 814 \mathrm{~W}}=28.8 \mathrm{mag}$. Their deep observations needed exposures of 39.1 hours in F606W and 45.5 hours F814W, spanning 120 orbits of HST/ACS imaging observations (Brown et al. 2003, 2004). In the future, such deep HST observations will only be obtained for a very small number of fields (e.g., Rich et al. 2005). As discussed in $\S 1$, Ma et al. (2007a) constrained the age of the M31 GC S312 by comparing multicolor photometry and theoretical stellar population synthesis models. It is encouraging that the age obtained by Ma et al. (2007a) is in good agreement with the previous determination based on main-sequence photometry (Brown et al. 2004), i.e., 9.5 $5_{-0.99}^{+1.15}$ Gyr versus $10_{-1}^{+2.5}$ Gyr. S312 is one of the few extragalactic GCs for which the age can be determined from main-sequence photometry. By comparing the ages of S312 and G1 determined using the same method, we can conclude that S312 is younger than the majority of the Galactic GCs at the same metallicity, and G1 is as old as the oldest Galactic GCs. In fact, if we try to fit the intrinsic SEDs of G1 by the theoretical BC03 SSP SEDs at an age of $10 \mathrm{Gyr}$, the resulting fit is very poor indeed, particularly in the ultraviolet. Therefore, we conclude that the method used in this paper, by which the ages of both S312 and G1 have been determined, can be used to determine the ages of old stellar populations to satisfactory precision, and in particular to distinguish between young and old populations.

\section{SUMMARY AND CONCLUSIONS}

In this paper, we first determined the age and mass of the M31 GC G1, as well as the realistic uncertainties associated with these estimates, by comparing its multicolor photometry with theoretical stellar population synthesis models. Our multicolor photometric data were obtained from GALEX FUV and NUV, broad-band optical $U B V R I$, and 2MASS $J H K_{\mathrm{S}}$ observations, which form an SED covering the wavelength range from 2267 to $20,000 \AA$. Our results confirm that G1 is one of the oldest and most massive GCs in the Local Group - that is, if it is indeed a genuine GC given that its mass is well in excess of the physical maximum mass predicted by the models of Gieles et al. (2006).

The age and mass obtained in this paper are somewhat dependent on the SSP model adopted. It is evident that the age of $18.23 \pm 1.76$ Gyr for G1 based on the BC03 models is greater than the currently accepted age of the Universe. However, we must keep in mind that the BC03 SSP models were calculated for ages up to $20 \mathrm{Gyr}$. In fact, ages derived for objects such as GCs and galaxies in excess of that of the Universe only mean that these objects are among the oldest objects in the Universe.

In the context of the BC03 models and their associated age range up to $20 \mathrm{Gyr}$, our derived age for G1 is consistent with the suggestion by the WMAP team that the oldest GCs may have formed within the first 1.7 Gyr after the Big Bang (see $§ 1)$.

The integrated FUV flux depends mainly on the fractional number of horizontal-branch (HB) stars with temperatures hotter than $T_{e} \sim 10,000 \mathrm{~K}$, with a modest dependence on their temperature distribution (see Rey et al. 2007 and references therein). Older GCs produce more of these hot HB stars and they are thus more likely to produce stronger FUV fluxes at a given metallicity (see Rey et al. 2007 and references therein) Lee et al. (2003) showed that the addition of FUV photometry to optical data can discriminate cleanly among young ( $<1$ Gyr), intermediate-age (3-5 Gyr), and old ( $>14$ Gyr) GCs. Young and very old GCs exhibit a significant FUV-to-optical spectral continuum slope, but intermediateage clusters are relatively faint in the FUV (see fig. 2 of Lee et al. 2003). Figure 6 of Rey et al. (2007) implies that the age of G1 may be similar to that of the oldest GCs.

Overall, we therefore conclude that G1 is indeed among the oldest and most massive building blocks of M31, and provides a key limitation to the age of the Universe, although we caution that our results also provide circumstantial support to the suggestion that the cluster may not be a genuine GC.

\section{ACKNOWLEDGMENTS}

We are indebted to the referee for thoughtful comments and insightful suggestions that improved this paper greatly. This work has been supported by the Chinese National Natural Science Foundation 
through Grant Nos. 10873016, 10803007, 10473012, 10573020, 10633020, 10673012, and 10603006; and by National Basic Research Program of China (973 Program) No. 2007CB815403. RdG acknowledges partial financial support from the Royal Society in the form of a UK-China International Joint Project. SCR acknowledges partial support from KOSEF through the Astrophysical Research Center for the Structure and Evolution of the Cosmos (ARCSEC). This paper makes use of data from the Two Micron All Sky Survey, which is a joint project of the University of Massachusetts and the Infrared Processing and Analysis Center, funded by NASA and the National Science Foundation. This paper is also partially based on archival observations with the NASA/ESA Hubble Space Telescope, obtained at the Space Telescope Science Institute (STScI), which is operated by the Association of Universities for Research in Astronomy, Inc., under NASA contract NAS 5-26555. This research has made use of NASA's Astrophysics Data System Abstract Service. This reasearch is partially based on archival data from the NASA GALEX mission developed in cooperation with the Centre National d'Etudes Spatiales of France and the Korean Ministry of Science and Technology.

\section{References}

Anders P., Bissantz N., Fritze-v. Alvensleben U., de Grijs R., 2004, MNRAS, 347, 196

Barmby P., Huchra J., Brodie J., Forbes D., Schroder L., Grillmair, C., 2000, AJ, 119, 727

Barmby P., Huchra J. P., 2000, ApJ, 531, L29

Barmby P., Huchra J. P., 2001, AJ, 122, 2458

Barmby P., Perrett K. M., Bridges T. J., 2002, MNRAS, 329, 461

Barmby P., McLaughlin D. E., Harris W. E., Harris G. L. H., Forbes D. A., 2007, AJ, 133, 2764

Bastian N., Gieles M., Lamers H. J. G. L. M., Scheepmaker R., de Grijs R., 2005,, A\&A, 431, 905

Bik A., Lamers H. J. G. L. M., Bastian N., Panagia N., Romaniello M., 2003, A\&A, 397, 473

Brown T. M. et al., 2003, ApJ, 592, L17

Brown T. M. et al., 2004, ApJ, 613, L125

Brodie J., Strader J., 2006, ARA\&A, 44, 193

Bruzual A. G., Charlot S., 1993, ApJ, 405, 538

Bruzual A. G., Charlot S., 1996, unpublished

Bruzual G., Charlot S., 2000, in Leitherer C. et al., eds, AAS CDROM Ser. Vol. 7. (Updated version of Bruzual G., Charlot S., 1996, PASP, 108, 996)

Bruzual A. G., Charlot S., 2003, MNRAS, 344, 1000 (BC03)

Cardelli J. A., Clayton G. C., Mathis J. S., 1989, ApJ, 345, 245

Cardiel N., Gorgas J., Sánchez-Blázquez P., Cenarro A. J., Pedraz S., Bruzual A. G., Klement J., 2003, A\&A, 409, 511

Carpenter J. M., 2001, AJ, 121, 2851

Carpenter J. M., Hillenbrand L. A., Skrutskie M. F., 2001, AJ, 121, 3160

Chaboyer B., Demarque P., Kernan P. J., Krauss L. M., 1998, ApJ, 494, 96

Chabrier G., 2003, PASP, 115, 763

Charlot S., Worthey G., Bressan A., 1996, ApJ, 457, 625

Cohen J. G., 2006, ApJ, 653, L21

de Grijs R., Bastian N., Lamers H. J. G. L. M., 2003a, MNRAS, 340, 197

de Grijs R., Fritze-v. Alvensleben U., Anders P., Gallagher J. S., Bastian N., Taylor V. A., Windhorst R. A., 2003b, MNRAS, 342, 259

de Grijs R., Anders P., Lynds R., Bastian N., Lamers H. J. G. L. M., O’Neill E. J. Jr., 2003c, MNRAS, 343, 1285

de Jong R. S., 1996, A\&A, 313, 377

Djorgovski S. G., Gal R. R., McCarthy J. K., Cohen J. G., de Carvalho R. R., Meylan G., Bendinelli O., Parmeggiani G., 1997, ApJ, 474, L19

Elias J. H., Frogel J. A., Matthews K., Neugebauer G., 1982, AJ, 87, 1029

Elias J. H., Frogel J. A., Hyland A. R., Jones T. J., 1983, AJ, 88, 1027

Fan Z., Ma J., de Grijs R., Yang Y., Zhou, X., 2006, MNRAS, 371, 1648 
Federici L., Bellazzini M., Galleti S., Fusi Pecci F., Buzzoni A., Parmeggiani G., 2007, A\&A, 473, 429 Frayn C. M., Gilmore G. F., 2002, MNRAS, 337, 445

Galleti S., Federici L., Bellazzini M., Fusi Pecci F., Macrina S., 2004, A\&A, 426, 917

Gieles M., Larsen S. S., Bastian N., Stein I. T., 2006, A\&A, 450, 129

Gratton R. G., Bragaglia A., Carretta E., Clementini G., Desidera S., Grundahl F., Lucatello S., 2003, A\&A, 408, 529

Gunn J. E., Griffin R. F., 1979, AJ, 84, 752

Gnedin O. Y., Ostriker J. P., 1997, ApJ, 474, 223

Goodwin S. P., 1997, MNRAS, 286, 669

Harris W. E., 1991, ARA\&A, 29, 543

Gebhardt K., Rich R. M. R., Ho, L. C., 2005, ApJ, 634, 1093

Holland S., Fahlman G. G., Richer H. B., 1997, AJ, 114, 1488

Huchra J. P., Brodie J. P., Kent S. M., 1991, ApJ, 370, 495

Kaviraj S., Rey S. C., Rich R. M., Yoon S. J., Yi S. K., 2007, MNRAS, 381, L74

Kim S. et al., 2007, AJ, 134, 706

King I., 1966, AJ, 71, 64

Kissler-Patig M., Brodie J. P., Minniti D., 2002, A\&A, 391, 441

Kroupa P., 2001, MNRAS, 322, 231

Landsman W., Bohlin R. C., Neff S. G., O'Connell R. W., Roberts M. S., Smith A. M., Stecher T. P., 1998, AJ, 116, 789

Lee H. C., Lee Y. W., Gibson B. K., 2003, in Extragalactic Globular Cluster Systems, ed. M. KisslerPatig (Berlin: Springer), 261

Leitherer C. et al., 1999, ApJS, 123, 3

Ma J., de Grijs R., Yang Y., Zhou X., Chen J., Jiang Z., Wu Z., Wu J., 2006, MNRAS, 368, 1443

Ma J. et al., 2007a, ApJ, 659, 359

Ma J. et al., 2007b, MNRAS, 376, 1621

MacArthur L. A., Courteau S., Bell E., Holtzman J. A., 2004, ApJS, 152, 175

Mayall N. U., Eggen O. J., 1953, PASP, 65, 24

Mengel S., Lehnert M. D., Thatte N., Genzel R., 2002, A\&A, 383, 137

Meylan G., Sarajedini A., Jablonka P., Djorgovski S. G., Bridges T., Rich R. M., 2001, AJ, 122, 830

Meylan G., 2002, in: Extragalactic Star Clusters, Geisler D., Grebel E. K., Minniti D., eds., (ASP: San Francisco), IAUS, 207, 555

O’Connell R. W., 1999, ARA\&A, 37, 603

Puzia T. H., Saglia R. P., Kissler-Patig M., Maraston C., Greggio L., Renzini A., Ortolani S., 2002a, A\&A, 395, 45

Puzia T. H., Zepf S. E., Kissler-Patig M., Hilker M., Minniti D., Goudfrooij P., 2002b, A\&A, 391, 453

Reed L. G., Harris G. L. H., Harris, W. E., 1994, AJ, 107, 555

Rey S. C. et al., 2005, ApJ, 619, L119

Rey S. C. et al., 2007, ApJS, 173, 643

Ribas I., Jordi C., Vilardell F., Fitzpatrick E. L., Hilditch R. W., Guinan E. F., 2005, ApJ, 635, L37

Rich R. M., Mighell K. J., Freedman W. L., Neill J. D., 1996, AJ, 111, 768

Rich R. M., Shara M. M., Zurek, D., 2001, AJ, 122, 842

Rich R. M., Corsi C. E., Cacciari C., Federici L., Fusi Pecci F., Djorgovski S. G., Freedman, W. L., 2005, AJ, 129, 2670

Romaniello M., 1998, Ph.D. Thesis, Scuola Normale Superiore di Pisa

Salpeter E. E., 1955, ApJ, 121, 161

Sarajedini A., 1994, AJ, 107, 618

Sarajedini A., Geisler D., Schommer R., Harding P., 2000, AJ, 120, 2437

Sargent W. L. W., Kowal C. T., Hartwic F. D. A., van den Bergh S., 1977, AJ, 82, 947

Scalo J. M., 1986, Fundamentals of Cosmic Physics, 11, 1

Schweizer F., Seitzer P., Brodie J. P., 2004, AJ, 128, 202

Searle L., Sargent W. L. W., Bagnuolo W. G., 1973, ApJ, 179, 427 
Schneider D. et al., 2002, AJ, 123, 458

Smith L. J., Gallagher J. S., 2001, MNRAS, 326, 1027

Sohn S. T., O’Connell R. W., Kundu A., Landsman W. B., Burstein D., Bohlin R. C., Frogel J. A., Rose J. A., 2006, AJ, 131, 866

Tinsley B. M., 1968, ApJ, 151, 547

Tinsley B. M., 1972, ApJ, 178, 319

VandenBerg D. A., Bergbusch P. A., Dowler P. D., 2006, ApJS, 162, 375

van den Bergh S., 1969, ApJS, 19, 145

Zinn R., West M. J., 1984, ApJS, 55, 45

Worthey G., 1994, ApJS, 95, 107

Wu H., Shao Z. Y., Mo H. J., Xia X. Y., Deng Z. G., 2005, ApJ, 622, 244 